\title{
Tendenzen und Inhalte unternehmensübergreifender Fabrikplanung
}

\author{
Dozent Dr-Ing. habil. Hans-Wolfgang Ahrend, Prof. Dr.-Ing. Claus-Gerold Grundig
}

\section{Problemstellung}

Die allgemein bekannte Planungsmethodik unternehmensbezogener Fabrikprozesse definiert Planungsphasen aufeinander aufbauender, unterschiedlichster Funktionsinhalte durch die eine stufenweise, systematisierte Projekterarbeitung und -realisierung methodisch strukturiert ist.

Typischer praktischer Einsatzfall der Fabrikplanungsmethodik ist immer dann gegeben, wenn komplexe Prozeßstufen der Logistik- und Wertschöpfungskette in der Entscheidungs- und Entwurfslogig „Funktionsbestimmung, Dimensionierung, Strukturierung und Gestaltung " zu konzipieren sind. Dabei sind konventionelle als auch rechnergestiitzte Vorgehensweisen im Planungsprozeß möglich - software tools visualisierter Fabrikplanung im Dialog insbesondere bei Einsatz von Simulations-, Entscheidungs- und Anordnungsbausteinen sind in Anwendung.

Neuere Aspekte z. B. ganzheitlicher Planung (Prozeßketten), integrierter Planung (Konstruktion simultan zu Layout) sowie des Logistikentwurfs werden verstärkt in die Planungssysteme eingebaut.

Eine kritische Betrachtung dieser Vorgehensweise in Verbindung mit aktuellen Tendenzen in der Industrie zeigt jedoch, daß eine weit durchgängigere, übergreifende und vernetzte Betrachtungsweise des Fabrikplanungsprozesses erforderlich ist. Dieser deutlich erweiterte Ansatz der Fabrikplanung hat von unternehmensuibergreifenden Strukturen und Inhalten auszugehen, so daß ganzheitliche Fabrikplanungsprozesse inhaltlich und methodisch zu strukturieren sind.

Nachfolgend werden ausgehend von Inhalten und Ablaufstrukturen der unternehmnsbezogenen Fabrikplanung einige wesentliche erweiterte Inhalte der unternehmensübergreifenden Fabrikplanungsmethodik dargestellt, die sich aus der erforderlichen ganzheitlichen Betrachtung der Prozeßkette ergeben. Ansätze zur Formalisierung methodischer Abläufe dieser deutlich erweiterten Fabrikplanungsmethodik werden aufgezeigt.

\section{Unternehmensbezogene Fabrikplanung - Vorreiter der unternehmensüber- greifenden Fabrikplanung}

Die in der industrieellen Anwendung praktizierten Fabrikplanungsmethoden gehen auf Planungsgrundsätze von ROCKSTROH, KETTNER, DOLEZALEK, AGGTELEKY und WIENDAHL zurïck. In Bild 1 ist in Anlehnung an KETTNER [1] der allgemeine Rahmenablauf der unternehmensbezogenen Fabrikplanungssystematik dargestellt [2].

\begin{tabular}{|c|c|}
\hline Planungsphase & Grundinhalte (Teilschritte) \\
\hline 1. Zielplanung & $\begin{array}{l}\text { - Projektidee } \\
\text { - Analyse: Ausgangslage } \\
\text { - Zielstellungen/Norgaben } \\
\text { - Aufgabenstellung (AST) }\end{array}$ \\
\hline 2. Vorarbeiten & $\begin{array}{l}\text { - Fabrikanalyse } \\
\text { - Planungsgrundlagen (Produktionsprogramm, Bedarf, } \\
\text { Aufwand) } \\
\text { - Vorentscheidung zum Losungsprinzip/Logistikentwurf } \\
\text { - Konkretisierung Aufgabenstellung (AST)/Entscheidung }\end{array}$ \\
\hline \multicolumn{2}{|l|}{ 3. Grobplanung } \\
\hline$\underline{3.1 \text { Idealplanung }}$ & 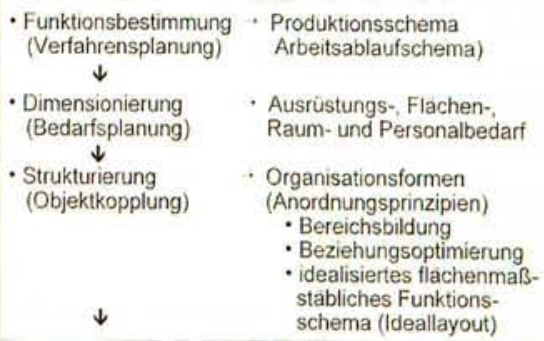 \\
\hline 3.2 Realplanung & 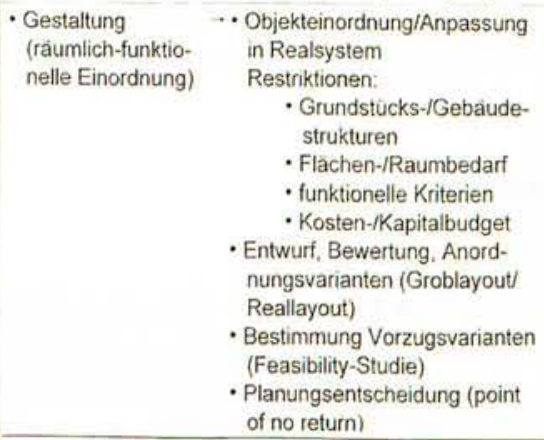 \\
\hline 4. Feinplanung & $\begin{array}{l}\text { Prázisierung Planungs- und Gestaltungsergebnisse } \\
\text { (Fein-Layout) } \\
\text { - Betriebsmittelanordnung (Fundamente/lnstallation) } \\
\text { - Zuordnung Ver- und Entsorgungstechniken } \\
\text { - Arbeitsplatzgestaltung (Abstände/Licht/Lärm/Arbeits- } \\
\text { schutz } \\
\text { - Feinabstimmungen Raum/Flache/Funktion } \\
\text { - Bauprojekt } \\
\text { - Genehmigungsverfahren } \\
\text { - Kontakte Liefer- und Ausführungsfirmen } \\
\text { - Erstellung Projektdokumentation (Ausführungsprojekt) }\end{array}$ \\
\hline$\frac{\text { 5. Ausführungs- }}{\text { planung }}$ & $\begin{array}{l}\text { - Uberprüfung Projektdokumentation } \\
\text { - Planung Bau-, Montage-, Installations-, Einrichtungs- } \\
\text { und Inbetriebnahmeablauf (Kapazitáten/Termine) } \\
\text { - Umzugspláne } \\
\text { - Bau- und Genehmigungsanträge } \\
\text { - Ausschreibungen/Angebote/Auftragsvergabe } \\
\text { - Festlegung Projektleitung/Projektmanagement } \\
\text { - Pflichtenhefte }\end{array}$ \\
\hline 6. Ausführung & $\begin{array}{l}\text { - Führung/Überwachung Projektrealisierung } \\
\text { (Projektleitung) } \\
\text { - Zwischen-/Funktionsprüfungen, Probelāufe } \\
\text { - Mitarbeitereinarbeitung } \\
\text { - Abnahmeprüfungen (Übergabe-/Inbetriebnahme- } \\
\text { protokolle) } \\
\text { - Ergànzung Projektdokumentation }\end{array}$ \\
\hline
\end{tabular}

Bikd 1: Systematisierter Rahmenablauf unternehmensbezogener Fabrikplanung (Grundprinzip) [2] 
Dieser ganzheitlich komplexen Betrachtungsweise folgend wurden objektbezogene, integrierte Fabrikplanungsinstrumente (Objektsoftware-pools) entwickelt (vgl. z.B. [3], [4]).

Wird die übliche Fabrikplanungspraxis kritisch betrachtet, zeigen sich oftmals beträchtliche Defizite insbesondere hinsichtlich des erzielten Grades der Durchgängigkeit im Sinne einer ganzheitlichen Planung. Verbreitet sind ungenügende Abstimmungen z.B. zwischen den Prozeßstufen Vorfertigung (VF) und Montage (M) infolge isolierter, zeitversetzter Planung. Auch werden Erfordernisse integrierter, zeitparalleler Planung der technischen Lösung der Ausführungsvariante (Planungsphase: Grob- und Ausfuihrungsplanung) mit der Organisationslösung (Logistik- und PPS-Prinzipien) nicht beachtet (vgl. auch Bild 1).

Negative Folgen für die Prozeßstufe M sind:

- verspätete Anlieferung von VF-Aufträgen

(Termindefizite, Montage- und Auslieferungsverzögerungen)

- ïberzogene Anlieferung von VF-Aufträgen

(Anwachsen Lagerbestände, Durchlaufzeiten)

- mangelhafte VF-Qualität

(Nacharbeit, Montageprozeßverzögerung)

Mengen- und Zeitdifferenzen zwischen VF-Ausstoß und Montagebedarf können z. B. durch fehlenden Losgrößenabgleich verursacht sein. Untersuchungen zeigen, daß oftmals VF-Losgrößen ausschließlich nach Prämissen der Prozeßstufe VF festgelegt werden und Montagebelange dabei unberüicksichtigt bleiben. Montagelosgrößen wiederum unterliegen in besonderem Maße den jeweils aktuellen Kundenauftragsstrukturen bzw. Kommissionierungsaspekten, so daß unterschiedliche, teils duale Optimierungsansätze zu ordnen sind. Weitere Einflußüberlagerungen ergeben sich durch Notwendigkeiten der logistikoptimalen PPS-Strategie, z. B. basierend auf dem KANBAN oder just-in-time-Prinzip.

Dem steht bekanntermaßen gegeniiber, daß alle Prozeßstufen einschließlich von Beschaffungs-, Lagerungs- und Vertriebsprozessen durchgängig - im Sinne einer unternehmensbezogenen Verbundfabrikplanung - zu planen sind, um ein wirtschaftliches Betreiben des Produktionssystems entlang der gesamten Prozeßkette zu sichern [5]. Unter Verbundfabrikplanung wird dabei die ganzheitliche, durchgängige Planung aller Logistik- und Wertschöpfungsstufen der Prozeßkette verstanden.

Die erforderliche Synchronisation von Flußsystemen unterschiedlicher Prozeßstufen erfordert prinzipiell:

- mengenmäßige Abstimmungen (Mengenabgleich)

- zeitliche Abstimmungen (Synchronisation von Anlieferungs- und Bedarfszeitpunkten, Definition von Mindest- und Maximalgrößen für Pufferbestände)

- qualitative Abstimmungen (Qualitätssicherungssysteme).

Werden die Synchronisations- und Pufferfunktionen von Lägern ausgeklammert (Lagervermeidung!) dann gilt, je flexibler das Leistungspotential der jeweils vorgelagerten Stufe, desto flexibler und wirtschaftlicher sind die kundendiktierten Bedarfserfordernisse der Folgestufe z. B. Montage - durchsetzbar.

Dementsprechend müssen z. B. Bereiche der VF folgendes Potential sichern:

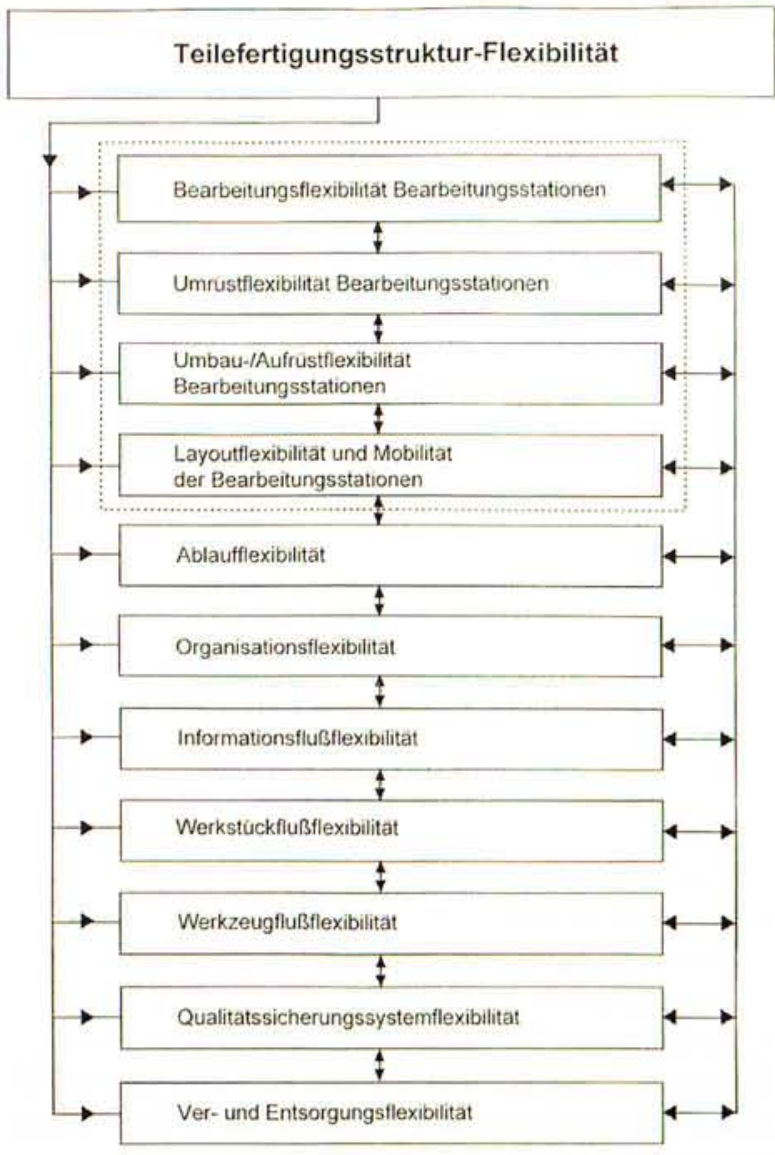

Bild 2: Teilefertigungsstrukturflexibilität und ihre Komponenten

- Minimale Durchlaufzeiten der Aufträge

- mengen- und zeitflexible Produktion unterschiedlichster Auftragsgrößen

- permanente Erzeugung der geforderten Werkstückqualität.

Die Durchsetzung dieser Zielaspekte macht eine technologisch und organisatorisch verursachte Strukturflexibilität von Bereichen der VF erforderlich, die - wie in Bild 2 dargestellt - in 11 Komponenten funktionell gegliedert werden kann, wobei die Flexibilitätskomponenten des Bearbeitungssystems die bestimmenden Kernkomponenten bilden.

Prinzipiell gilt, die Flexibilität der VF-Struktur ist um so zwingender, je kleiner die zu fertigenden VF-Losgrößen $(->$ Losgröße 1) sind bzw. je turbulenter die Marktdynamik auf den Bereich durchschlägt.

Nur im „harmonischen“ Zusammenwirken aller 11 Komponenten kann die VF-Flexibilität voll wirksam werden. Bezogen auf das Bearbeitungssystem wird damit ein hohes Automatisierungsniveau unerläßlich, wenn die Flexibilitätsforderungen seitens der Montage bzw. des Marktes hoch sind.

Steigendes Automatisierungsniveau wiederum ermöglicht in Verbindung mit dem Integrationspaket mehrfache Effekte - wie aus Bild 3 ersichtlich ist.

Das mögliche Automatisierungsniveau von Bearbeitungsstationen ist in Tafel 1, in Form von 6 möglichen Automatisierungsstufen dargestellt. Dabei gilt, je höher die Automatisierungsstufe der Bearbeitungsstationen ist, desto höher sind Flexibilität und Produktivität. Erzielbare Effekte hoher Bearbeitungsstationenflexibilität sind: 


\section{Grundtendenzen}

Automatisierung und Integration

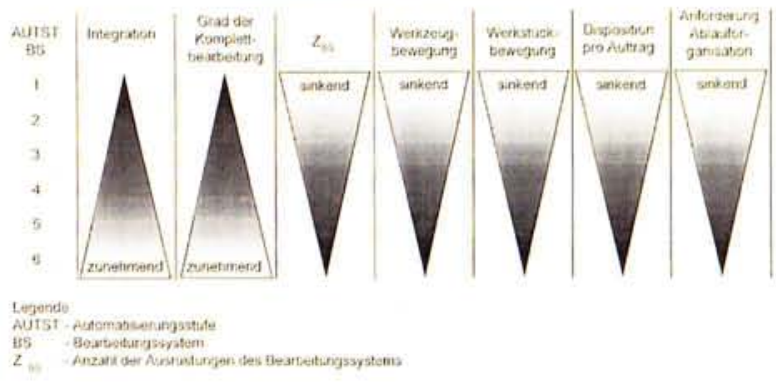

Bild 3: Effekte der Automatisierung und Integration Bearbeitungsstationen

- große technologische Spannweite der bearbeitbaren Teilespektren (Werkstiucksortimente)

- Realisierbarkeit minimaler Losgrößen (bis 1) bei wirtschaftlicher Fertigung beliebiger Auftragsreihenfolgen

- schnelles Reagieren auf aktuell wechselnde Bedarfe (Umdispositionen, Eilläufer)

- Termintreue und hohe Lieferfähigkeit (Durchsatz)

- Senkung Lager- und Kapitalbindungskosten als Folge minimierter Lagerung

- Senkung von Durchlauf- und Lieferzeiten.

Vorgenannte Effekte entsprechen wesentlichen Globalzielen der Fabrikplanung - insbesondere der Produktionslogistik.

In Ansätzen sollte aufgezeigt werden, daß diese (unternehmensbezogene) Verbundfabrikplanung erforderlich ist, z.B. zur Sicherung der zeit-, mengen- und qualitätssynchronen Produktion unterschiedlicher Prozeßstufen. In der industriellen Anwendung dieser Verbundfabrikplanung wurden eine Vielzahl von Erfahrungen gesammelt. Diese sind gezielt auf die Inhalte der unternehmensübergreifenden Fabrikplanung durch Adaption auf die veränderten Rahmenbedingungen anzupassen, so

\begin{tabular}{|c|c|}
\hline AUTST $_{i}$ & Beschreibung \\
\hline 1 & konventionelle WZM \\
\hline 2 & NC-WZM \\
\hline 3 & CNC-WZM \\
\hline 4 & NC-BAZ \\
\hline 5 & CNC-BAZ \\
\hline 6 & WZM, BAZ im DNC-Betrieb \\
\hline \multicolumn{2}{|l|}{ Legende: } \\
\hline $\begin{array}{l}\text { AUTST, } \\
\text { BAZ } \\
\text { WZM } \\
i\end{array}$ & $\begin{array}{l}\text { - Automatisierungsstufe I } \\
\text { - Bearbeitungszentrum } \\
\text { - Werkzeugmaschine } \\
\text { - Laufvariable }\end{array}$ \\
\hline \multicolumn{2}{|c|}{ Anmerkung. } \\
\hline \multicolumn{2}{|c|}{$\begin{array}{l}\text { Neue Werkzeugmaschinen-Grundtypen sind problemlos in die } \\
\text { Tabelle einzuordnen. }\end{array}$} \\
\hline \multicolumn{2}{|c|}{$\begin{array}{l}\text { WZM-Grundtypen mit gleicher Leistungsfahigkeit einer der fixierten } \\
\text { Automatisierungsstufen ist oben in dieser Aut-Stufe einzuordnen. }\end{array}$} \\
\hline \multicolumn{2}{|c|}{$\begin{array}{l}\text { WZM-Grundtypen mit hoherer Leistungsfahigkeit als die AUTST } 6 \\
\text { erweitern die Tabelle - AUTST } 7\end{array}$} \\
\hline
\end{tabular}

Tafel 1: Automatisierungsstufen - Bearbeitungssituationen daß der Neueinstieg in Abläufe und Inhalte einer unternehmensuibergreifenden Fabrikplanungssystematik determiniert ist.

\section{Unternehmensübergreifende Fabrikplanung}

Die unternehmensübergreifende Verbundfabrikplanung ist in 3 grundsätzliche Objektverbunde gliederbar:

- Zulieferer (Vorgänger) $\longrightarrow$ Finalproduzent

- Finalproduzent $\longrightarrow$ Verbraucher, Abnehmer (Recycling, Entsorgung)

- Zulieferer (Vorgänger) $\longrightarrow>$ Finalproduzent $\longrightarrow>$ Verbraucher, Abnehmer

Die durchgängige Prozeßkette der Produkterstellung und des -verbrauches kann folglich auf territorial (länderübergreifend) unterschiedlichste Unternehmen bzw. Unternehmensbereiche aufgesplittet sein, wobei dann netzartig flexibel kooperierende Unternehmensstrukturen typisch sind.

Aus fabrikplanerischer Sicht wird damit eine unternehmensübergreifende, die gesamte Prozeßkette des Produktzyklus zu grunde legende Fabrikplanung aller beteiligten Bereiche bzw. Unternehmen erforderlich (unternehmensübergreifende Verbundfabrikplanung). Dabei werden die im Bild 4 dargestellten 3 Objektbereiche und 2 Objektverbunde relevant:

- Objektbereich I - nur 1 Prozeßstufe wird jeweils separat geplant, benachbarte Prozeßstufen werden kaum berücksichtigt

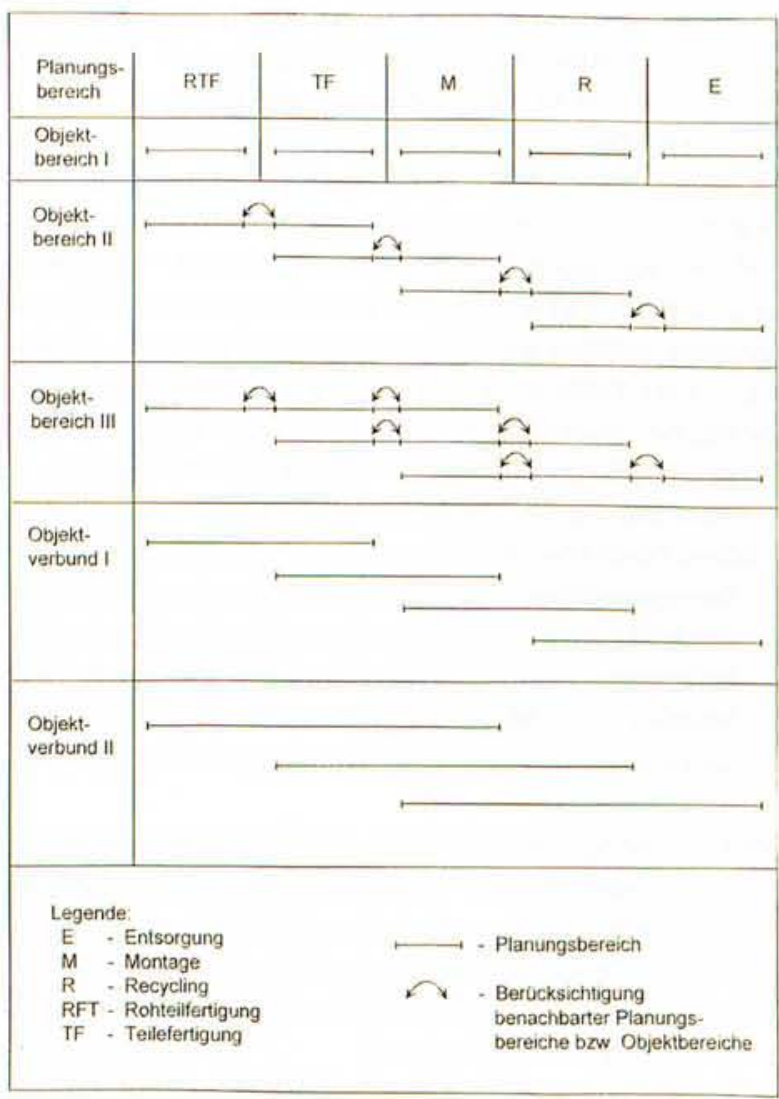

Bild 4: Objektbereiche und Objektverbunde der Fabrikplanung 
- Objektbereich II - zwei Prozeßstufen werden jeweils separat, aber parallel (ganzheitlich) geplant, benachbarte Prozeßstufen werden global (im Block) berüicksichtigt

- Objektbereich III - drei Prozeßstufen werden jeweils separat, aber parallel (ganzheitlich) geplant, benachbarte Prozeßstufen werden global (im Block) berüicksichtigt

- Objektverbund I - 2 benachbarte Prozeßstufen werden im Verbund geplant

- Objektverbund II - 3 benachbarte Prozeßstufen werden im Verbund geplant

Die in Bild 4 dargestellten Objektbereiche und -verbunde sind fallorientiert anpaßbar bzw. erweiterungsfähig. Die Umsetzung dieser unternehmensübergreifenden Fabrikplanung wird immer zwingender - auch unter den speziellen Aspekten der Sicherung durchgängiger wirtschaftlicher Logistikketten (vgl. auch [6]).

Ursachen dieser Entwicklung sind - Globalisierung der Produktionsstandorte bei (im Regelfall) Abnahme der Fertigungstiefe des Finalproduzenten, auch unter den Aspekten kostengünstiger Produktion, bestandsarmer Fertigung, Konzentration auf Kernkompetenzen weiterhin Zwänge zum wirtschaftlichen Recycling und zur weiteren Minimierung der Entsorgung von Restkomponenten. Aber auch die Folgen turbulenter Märkte, regional verstärkter Kostendruck, die kooperative Nutzung von Potentialen (z. B. in Formen von Produktionsnetzen [7]) erfordern eine ketten- und netzorientierte unternehmensübergreifende Fabrikplanung.

Industriesituation ist, daß z. B. der europäische Automobilbau nur noch eine Fertigungstiefe von 35-38\%, der japanische nur noch von 20-30\% aufweist [8]. Abnehmende Fertigungstiefen verstärken die Kooperationsbeziehungen netzartig zwischen dem Finalproduzenten und seinen Zulieferern enorm. Diese Zuliefererbeziehungen entwickeln sich dabei zu Wertschöpfungspartnerschaften. Damit wird die unternehmensübergreifende Verbundfabrikplanung der beteiligten Partner unerläßlich aber auch praktikabel. Die Fabrikstrukturen der Wertschöpfungspartner auf der Zulieferseite müssen mit den Fabrikstrukturen des Finalisten so harmonieren, daß die Zulieferteile qualitäts-, sortenund termingerecht in den Produktionsprozeß des Finalproduzenten (Folgestufe) logistikgerecht eingeschleust werden können. So werden z.B. durch Anwendung von Prinzipien der just-in-time-Produktion in der Logistikkette zwischen Zulieferer und Finalproduzent radikale Lieferzeit-, Bestands- und Logistikaufwandsminimierungen erzielt, Eingangskontrollen und Zwischenlagerungen bei Finalisten können entfallen. Fabrikstrukturen (bereiche) des Zulieferers und des Abnehmers sind wie unmittelbar „benachbarte“ Prozeßstufen zu konzipieren wobei die oftmals räumliche Entkopplung der Partner durch das Logistikelement „aufwandsarmer, umweltschonender Transport" einschließlich entsprechender Informationstechnik zu überbrüicken ist. Deutlich wird damit die Notwendigkeit einer Neuordnung von Fabrikstrukturen einschließlich der Fabrikorganisation - aus methodischer Sicht wird dafür ein systematisch-ganzheitliches Planungskonzept mit wesentlich erweiterten Inhalten gegenüber Bild 1 erforderlich.

Dabei sind eine Vielzahl, teils gegenläufiger Einflüsse zu beachten. So kann durch die eingetretene Entwicklung von Zulieferern zu kompetenten Systemlieferanten den negativen Auswirkungen (wie z. B. erhöhtes Verkehrsaufkommen, zusätzliche Umweltbelastungen) abnehmender Fertigungstiefe beim Abnehmer entgegengewirkt werden. Dieser Problemkomplex ist in Bild 5 anschaulich dargestellt [9] wobei als Zulieferer der Vorfertiger (z. B. Teilefertiger) gewählt ist.

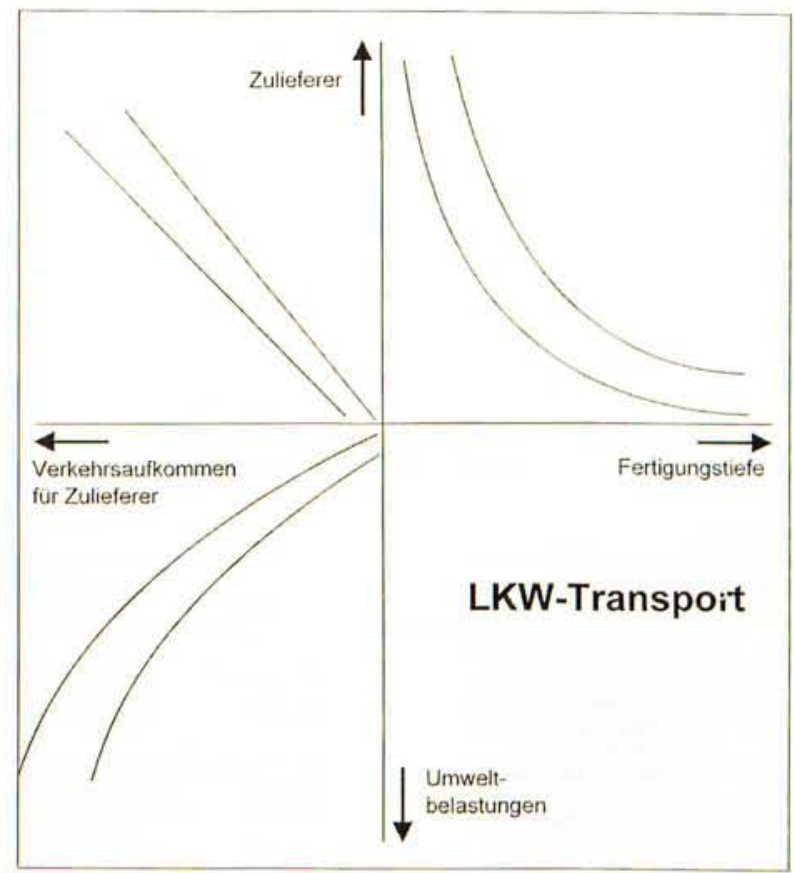

Bild 5: Qualitativer Zusammenhang Unternehmensfertigungstiefe Verkehrsaufkommen - Umweltbelastung [9]

Nach ABERLE ist die tägliche Verkehrsdichte (Fahrzeuge/ 24 h) von 1960 bis 1990 signifikant gestiegen:

- Bundesautobahn auf $390,7 \% 41.800 \mathrm{FZ} / 24 \mathrm{~h}$

- Bundesstraßen auf 253,8\% 9.010 FZ/24 h

- Landesstraßen auf $279,7 \% 3.530 \mathrm{FZ} / 24 \mathrm{~h}$

- Kreisstraßen auf $365,3 \% 1.655$ FZ/24 h.

Diesen Entwicklungen entsprechend ergeben sich sehr differenzierte, strategisch globale Vorgehensweisen zur Entwicklung regional kernstandortbezogener Kooperationsnetze im Unterschied zu solchen, die weltweit strukturiert sind. Deutlich werdende ökologische und soziale Probleme sind in die unternehmensübergreifende Fabrikplanung einzubeziehen.

Tendenziell können drei Ausprägungsformen hinsichtlich der Komplexität der Fabrikplanung unterschieden werden:

1. Rein unternehmensbezogene Fabrikplanung (Planungssystematik nach Bild 1) - Komplettfertiger hoher Fertigungstiefe

2. Unternehmensbezogene Fabrikplanung - allerdings mit Globalabstimmungen zwischen kooperierenden Partnern (Planungssystematik nach Bild 1) - begrenzte Fertigungstiefe. 


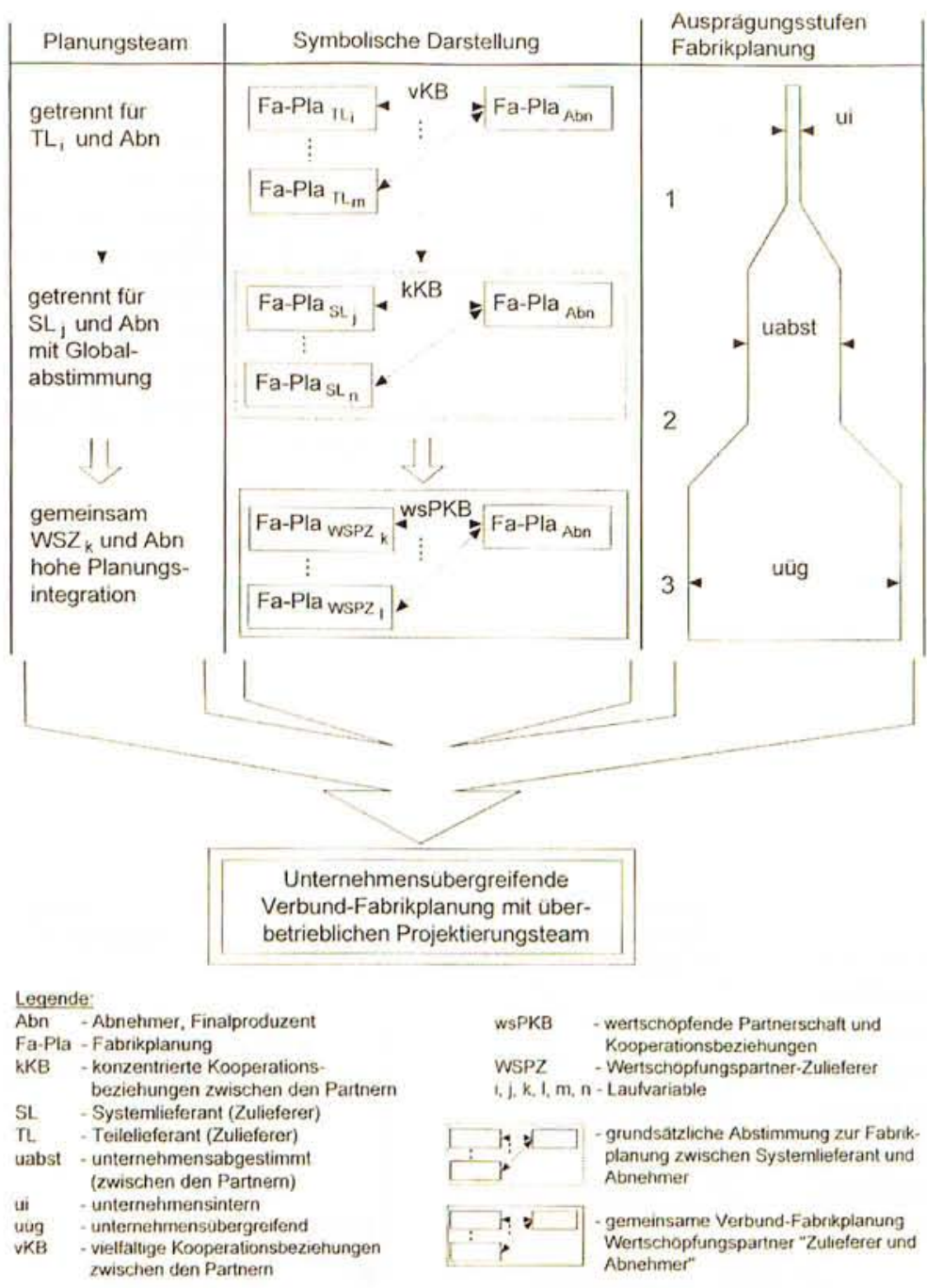

Bild 6: Unternehmensübergreifende Verbundfabrikplanung (Beispiel Zulieferer-Abnehmer)

Bild1) unter Einbeziehung von Fachkräften der Konstruktion und Arbeitsvorbereitung entsprechende Strukturformen aufzubauen. Kritiklose „unbesehene“ Einordnung von Produkten in Fabrikprozesse ist zu vermeiden - zwei prinzipielle Sachverhalte sind hierbei zu unterscheiden:

1. Abfallbeseitigung, die sich aus dem Herstellungsprozeß ergibt.

2. Abfallbeseitigung, die sich nach dem Produktgebrauchsende ergibt.

Die erste Abfallkategorie ist für die unmittelbare Fabrikplanung der Herstellungsprozesse relevant. Hieraus folgt, daß die Prinzipien der Abfallvermeidung durch Konzipierung von abfallarmen/abfallfreien Fabrikstrukturen durchzusetzen sind, so z. B. durch Prozesse Präzisionsschmieden, Präzisionsgießen, stufenarme Prozesse, aber auch durch solche Konstruktionslösungen, bei denen das Rohteilvolumen maximal dem Fertigteilvolumen angenähert ist. Diese Entwicklung bestimmt maßgeblich die Auswahl von Betriebsmitteln für technologische Prozesse und damit die Fabrikstruktur des Herstellungsprozesses. Aber auch die Fabrikgestaltung der entsprechenden Recycling- und Entsorgungsbereiche wird positiv beeinflußt.

Die zweite Abfallkategorie wirkt unmittelbar im Recycling-Entsorge-Bereich. Der Fabrikplaner ist so zu „motivieren“ (Ge-

3. Unternehmensübergreifende (gemeinsame) Fabrikplanung kooperierender Wertschöpfungspartner Finalproduzent bei minimierter Fertigungstiefe, Konzentration auf Kernkompetenzen.

Die Aktualität unternehmensübergreifender Fabrikplanung wird auch deutlich durch die Notwendigkeit der Gestaltung der Finalproduzent-Nachfolger(Abnehmer/ Verbraucher)-Beziehungen. Bei diesen Beziehungen sind die Recycling- und Entsorgungserfordernisse von groBer Aktualität [10].

Hohe Maßstäbe werden durch das neue Kreislaufwirtschafts-/Abfallgesetz (KrW/ AbfG) gesetzt.

Die bisherige Prioritätenfolge der Abfallbehandlung gilt weiterhin in der Wichtung:

- Abfall vermeiden

- Abfall vermindern

- Abfall verwerten

- Abfall (Restkomponenten) gemeinwohlverträglich beseitigen/entsorgen

Zur Vermeidung von Abfall ist eine abfallfreie/-arme Produkt- und Prozeßgestaltung unerläßlich. Der Fabrikplaner hat folglich bereits in der „Entwurfsphase“ von Fabrikstrukturen (z. B. Planungsphase „Vorarbeiten“ in setzgebung Produktverwertung), schon zum Zeitpunkt des Entwurfs der Fabrikstruktur für den Herstellungsprozeß bereits gleichzeitig den zeitlich versetzt oftmals wesentlich später stattfindenden Recycling- und Entsorgungsprozeß hinsichtlich seiner Fabrikstruktur zu entwerfen - auch um Aufwände zu erkennen u.U. mit Konsequenzen auf die Konstruktionslösung. Die Phasenverschiebung zwischen Herstellung und Recycling/Entsorgung ist erzeugnisabhängig unterschiedlich:
weiße Ware
-5 bis 6 Jahre
braune Ware
-6 bis 8 Jahre
Personenkraftwagen - 10 bis 12 Jahre.

Werden die Erfordernisse der Abfallvermeidung und wirtschaftlichen Abfallbeseitigung entsprechend dem Kreislaufwirtschafts- und Abfallgesetz beachtet, ergibt sich automatisch die Einbeziehung der FinalproduzentNachfolger-Beziehung im Sinne einer unternehmensübergreifenden Verbundfabrikplanung unter Einbindung der Recycling- und Entsorgungsprozesse. Oftmals sind Finalproduzent, Recyclingbetrieb und Entsorgungsbetrieb eigenständige Unternehmen. Damit ergibt sich auch hier die Verbundfabrikplanung mit unternehmensübergreifendem Charakter einschließlich der Besonderheit von zeitlicher Phasenverschiebung der Herstellungs- und Recyclingprozesse. Bei Recyclingprozessen 
ist zwischen Produkt- und Materialrecycling zu unterscheiden. Produktrecycling hat Vorrang vor Materialrecycling und wird maßgeblich von der realisierbaren Demontageart bestimmt. Die „Demontagefreundlichkeit" der Produkte ist gegeben, wenn:

- die Verbindungen des Erzeugnisses leicht lösbar und gut zugänglich sind

- Beschädigungen durch Demontage an wiederverwendbaren Erzeugnissen und Teilen nicht auftreten

- eine hohe Wiederverwendungsfähigkeit bzw. Aufarbeitungsfähigkeit möglichst vieler Demontageelemente gegeben ist.

Daraus folgt, eine möglichst zerstörungsfreie Demontage ist anzustreben, die zerstörende Demontage hingegen ist demontageunfreundlich. Nach EVERSHEIM [11] kann mittels eines Demontage-Bewertungsverfahrens die Montagegerechtheitskennzahl DGKB (für Baugruppen) ermittelt werden. Bei DGKB $=1$ liegt die maximale Demontagegerechtheit vor (vgl. Tafel 2).

\begin{tabular}{|c|c|c|c|c|c|c|c|}
\hline \multirow{2}{*}{\multicolumn{2}{|c|}{ Bewertungsknterium }} & \multirow{2}{*}{$\begin{array}{c}\text { Gewich- } \\
\text { tung } \\
{[\mathrm{G}]}\end{array}$} & \multicolumn{2}{|l|}{ Unterkriterien } & \multicolumn{2}{|l|}{ Punkte } & \multirow{2}{*}{$\begin{array}{l}\text { DGK }_{E(1)} \\
=P^{P(1)} \\
P_{\max }(1)\end{array}$} \\
\hline & & & Kriterium & {$\left[u_{i}\right]$} & \begin{tabular}{|l|}
$P_{\max }=$ \\
$G^{*} u_{i \max }$
\end{tabular} & $\begin{array}{l}P= \\
G^{*} u_{i}\end{array}$ & \\
\hline \multicolumn{2}{|c|}{ 1) Zerlegungsrichtungen } & 10,000 & $\begin{array}{l}\text { von oben } \\
\text { seilkcil } \\
\text { von unten }\end{array}$ & $\begin{array}{l}5 \\
3 \\
1\end{array}$ & 50,000 & & \\
\hline \multicolumn{2}{|c|}{$\begin{array}{l}\text { 2) Anzahl gemeinsamer } \\
\text { Bauelemente in } \\
\text { mehreren Baugruppen }\end{array}$} & 8.750 & $\begin{array}{c}\text { keins } \\
1 \\
2 \\
3 \\
\text { menr als } 3\end{array}$ & $\begin{array}{l}5 \\
4 \\
3 \\
2 \\
1\end{array}$ & 43,750 & & \\
\hline \multicolumn{2}{|c|}{$\begin{array}{l}\text { 3) Separate Verbin- } \\
\text { dungselemente }\end{array}$} & 8.125 & $\begin{array}{c}\text { keins } \\
1 \\
2 \\
3 \\
\text { menf als } 3 \\
\end{array}$ & $\begin{array}{l}5 \\
4 \\
3 \\
2 \\
1 \\
\end{array}$ & 40,625 & & \\
\hline \multicolumn{2}{|c|}{$\begin{array}{l}\text { 4) Anzahl Verbindungs- } \\
\text { verfahren }\end{array}$} & 8,125 & $\begin{array}{c}1 \\
2 \\
3 \\
\text { mehr als } 3\end{array}$ & $\begin{array}{c}5 \\
3.7 \\
2.4 \\
1\end{array}$ & 40,625 & & \\
\hline \multicolumn{2}{|c|}{$\begin{array}{l}\text { 5) Zerlegungs- } \\
\text { bewegungen }\end{array}$} & 7,500 & $\begin{array}{l}\text { linear } \\
\text { rotalonisch } \\
\text { kombinient }\end{array}$ & $\begin{array}{l}5 \\
3 \\
1\end{array}$ & 37,500 & & \\
\hline \multicolumn{2}{|c|}{ 6) Autornatisierbarkeit } & 7,500 & $\begin{array}{l}\text { lineat } \\
\text { tolatorisch } \\
\text { kombinient }\end{array}$ & $\begin{array}{l}5 \\
3 \\
1\end{array}$ & 37.500 & & \\
\hline \multicolumn{2}{|c|}{ 7) Hilfsmittel } & 6.875 & $\begin{array}{l}\text { keane } \\
\text { allround } \\
\text { spezielle }\end{array}$ & $\begin{array}{l}5 \\
3 \\
1\end{array}$ & 34,375 & & \\
\hline \multicolumn{2}{|c|}{ 8) Steifigkeit } & 6.250 & $\begin{array}{c}\text { formstabil } \\
\text { bedingt formstabil } \\
\text { biegeschlatt }\end{array}$ & $\begin{array}{l}5 \\
3 \\
1\end{array}$ & 31,250 & & \\
\hline 9) Anz: & ahl Fügeflachen & 6.250 & $\begin{array}{l}1 \\
2 \\
3\end{array}$ & $\begin{array}{r}5 \\
3.7 \\
2.4 \\
\end{array}$ & 31,250 & & \\
\hline \multicolumn{8}{|c|}{ Legende } \\
\hline $\begin{array}{l}\text { B } \\
\text { DGK } \\
\text { E } \\
G \\
i \\
P \\
u\end{array}$ & $\begin{array}{l}\text { - Baugruppenbewe } \\
\text { - Demontage-Gere } \\
\text { - Einzelmerkmalbe } \\
\text { - Gewichtungsfakto } \\
\text { - Laufvariable für B } \\
\text { - Punktzahl fur Ken } \\
\text { - Multiplikator eine }\end{array}$ & $\begin{array}{l}\text { ertung } \\
\text { echtheits-k } \\
\text { ewertung } \\
\text { lor } \\
\text { Bauteile } \\
\text { nnzahibild } \\
\text { Unterkrite }\end{array}$ & Cennzaht & & \multicolumn{3}{|c|}{$\frac{\sum P_{1}}{\Sigma P_{1}}$} \\
\hline
\end{tabular}

Tafel 2: Demontagegerechtigkeitskennzahl DGB [11]

Die Demontagegerechtheit der Produkte bestimmt maßgeblich das realisierbare Automatisierungsniveau im Demontageprozeß. Wirtschaftliche Aspekte sind allerdings bei der Fixierung des sinnvollen Automatisierungsniveaus ausschlaggebend, dabei werden folgende Orientierungswerte aus der Sicht der Demontagegerechtheit empfohlen:

$\mathrm{O}<$ DGK $<0,4 \longrightarrow$ manuelle Demontage

$0,5<$ DGK $<0,7 \rightarrow$ flexible automatisierte Demontage $0,8<$ DGK $<1 \rightarrow$ stark automatisierte Demontage.
Weitere Einflüsse auf das realisierbare Automatisierungsniveau der Demontage sind:

- die zu demontierenden Stückzahlen

- Typenreinheit/ Typenvielfalt der zu demontierenden Erzeugnisse

- Art der verwendeten Füge- und Verbindungstechniken im Montageprozeß

- die entstehenden Demontagezeitaufwände

- Restriktionen zur Standortfindung der Demontage

- Personaleinsatz im Demontageprozeß

- Weiterverwendbarkeit demontierter Baugruppen und Einzelteile (Ersatzteilverkauf, Aufarbeitung, Materialrecycling).

Im Falle erforderlicher Entsorgung von Restkomponenten gelten für die Fabrikplanung der Entsorgungsprozesse folgende Prämissen:

- geringstmögliche Inanspruchnahme von Deponieraum

- biologische Abbaubarkeit bevorzugen

- Entsorgung von Asche und sonstigen Rüickständenbei thermischer Verwertung

- Minimierung der Sondermüllkomponenten.

Entsorgung bildet hierbei den letzten Ausweg im ökologischen Produktlebenszyklus. Dabei ist von einer stetigen Verknappung verfuigbarer Deponieräume auszugehen. Entsorgungskonzepte der Fabrikplanung müssen beachten, daß sich Industrie-Müllstrome im Regelfall ab speziellen Zeitpunkten mit Haus-Müllströmen vereinigen.

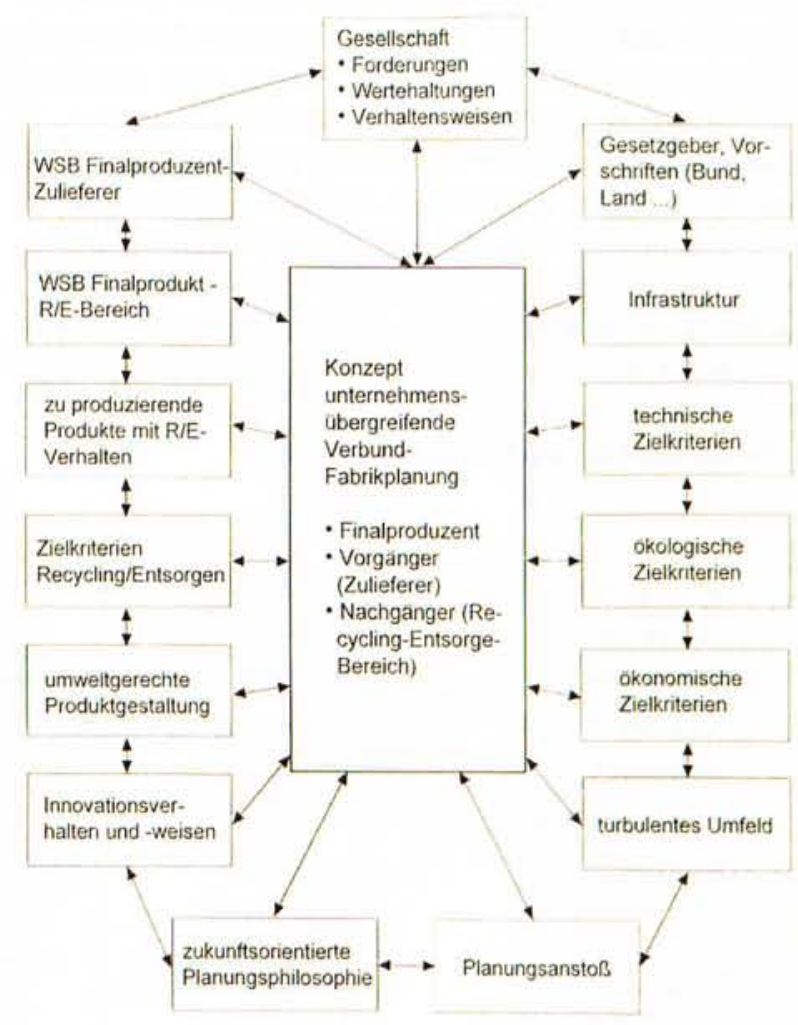

$$
\begin{aligned}
& \text { Legende } \\
& \text { E - Entsorgen } \\
& R \quad \text { - Recycling } \\
& \text { WSB } \\
& \text { - Wertschopfungsbeziehungen }
\end{aligned}
$$

Bild 7: Wesentliche Einflußkomplexe auf die unternehmensübergreifende Verbund-Fabrikplamung 


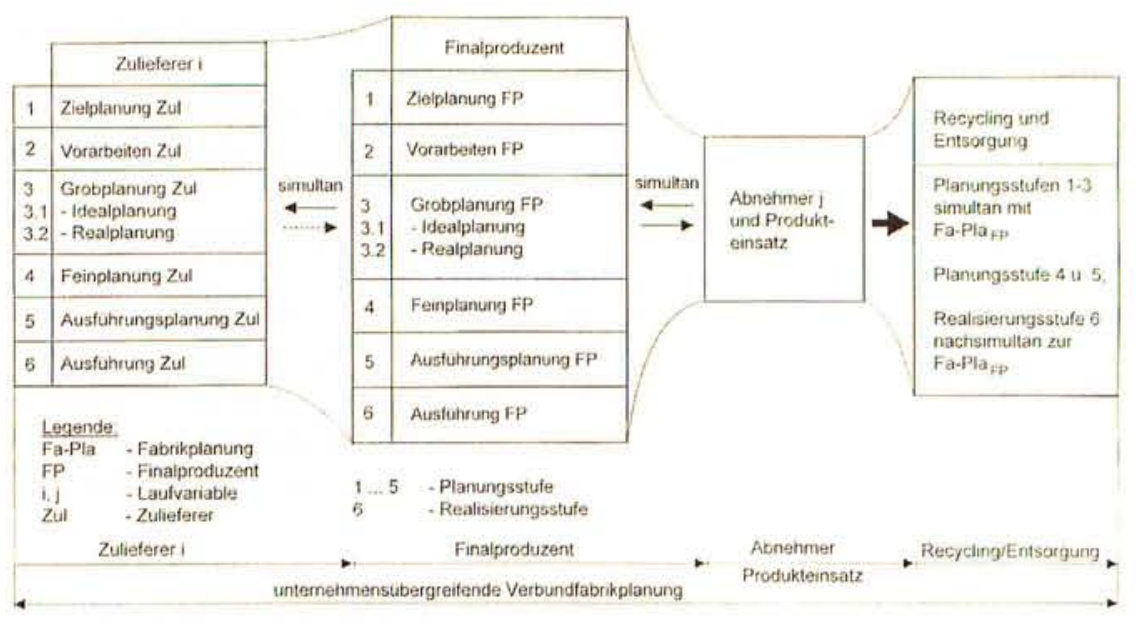

Bild 8: Unternehmensübergreifende Verbundfabrikplanung - Zulieferer-Finalproduzent - Abnehmer Recycling/Entsorge - Beziehungen

Deutlich wurde, Konzepte einer unternehmensübergreifenden Verbund-Fabrikplanung werden von einer Vielzahl unterschiedlichsterEinflußkomplexe geprägt wie in Bild 7 dargestellt. Die Wirkungen der Einflußkomplexe können gleichgerichtet aber auch entgegengesetzt sein. Die Aufgaben des übergreifenden Fabrikplanungsteams bestehen zunächst darin, strategische Grundentscheide zur durchgängigen Fabrikstruktur im Rahmen des Zielkriterien-Komplexes zu treffen. Diese Vorgehensweise verkörpert eine Neuorientierung der Fabrikplanungsphilosophie zu einer umfassenderen unternehmensüibergreifenden Fabrikplanung. Evolutionäre Entwicklungen hinsichtlich Komplexität und Inhalten der Fabrikplanung werden erkennbar, die ihre entsprechende methodische Umsetzung in einer erweiterten Fabrikplanungssystematik erfordern.

Dominante, erweiterte Inhalte sind die Durchgängigkeit der Prozeßkette in Form von Zulieferer (Vorgänger)

$\rightarrow$ Finalproduzent $\rightarrow>$ Nachfolger (Verbraucher/ Recycling/ Entsorgung). Dabei können die zeitlichen Phasenverschiebungen zwischen dem Herstellungs- und Recycling- bzw. Entsorgungsprozeß sehr kurz sein -desto dringlicher wird die Umsetzung einer unternehmensübergreifenden Verbundfabrikplanung.

Ausgehend vom Bild 1 sind in Bild 8 für die Bereiche Zulieferer sowie Recycling/Entsorgung die 6 Planungsphasen in ihrer grundsätzlichen Ablauffolge konzipiert.

Aus Gründen der Phasenverschiebung zwischen Herstellungsprozeß einerseits und dem Recycling/Entsorgungsprozeß andererseits ist der Planungsablauf (6 Phasenmodell) zeitlich so zu differenzieren, daß in einer ersten Etappe unmittelbar simultan zum Herstellungsprozeß die Phasen 1 bis 3 für Recycling/ Entsorgungsprozesse abzuarbeiten sind. In einer zweiten Etappe werden dann die Phasen 5 und 6 zeitversetzt durchgeführt. Dieser Planungsbeginn wird wesentlich von Kriterien des Produktlebenszyklus bzw. der Außerbetriebnahme der Produkte zeitlich bestimmt - vgl. Bild 9.

\section{Zusammenfassung}

Planungshorizont (Vorlauf) und Planungsumfang (Durchgängigkeit, Komplexität) der Fabrikplanung erweitern sich beträchtlich. Fabrikplanung im Netz kooperierender Unternehmen klärt schon in früher Phase die Vorgänger-Finalproduzent-Nachfolger-Beziehungen ab. In dieser Beziehungskette besitzen natürlich die Vorgänger-Finalproduzent-Beziehungen andere Wesensmerkmale als die Finalproduzent-Nachfolger-Beziehungen. Bei den Zulieferer-Abnehmer-Beziehungen sind die Abnehmer-Fertigungstiefe und der Zuliefertyp (z. B. Systemlieferant) prägende Einflußkomplexe. Die umzusetzenden Recycling- und Entsorgungsziele sind bei den Finalproduzent-Nachfolger-Beziehungen dominant. Die Breite des Aufgabenspektrums „Herstellung - Recycling - Entsorgung “ ist im Regelfall nur durch mehrere eigenständige Unternehmen abdeckbar. Hieraus folgt, die Verbundfabrikpklanung ist unternehmensübergreifend zu organisieren. Nur überbetriebli-

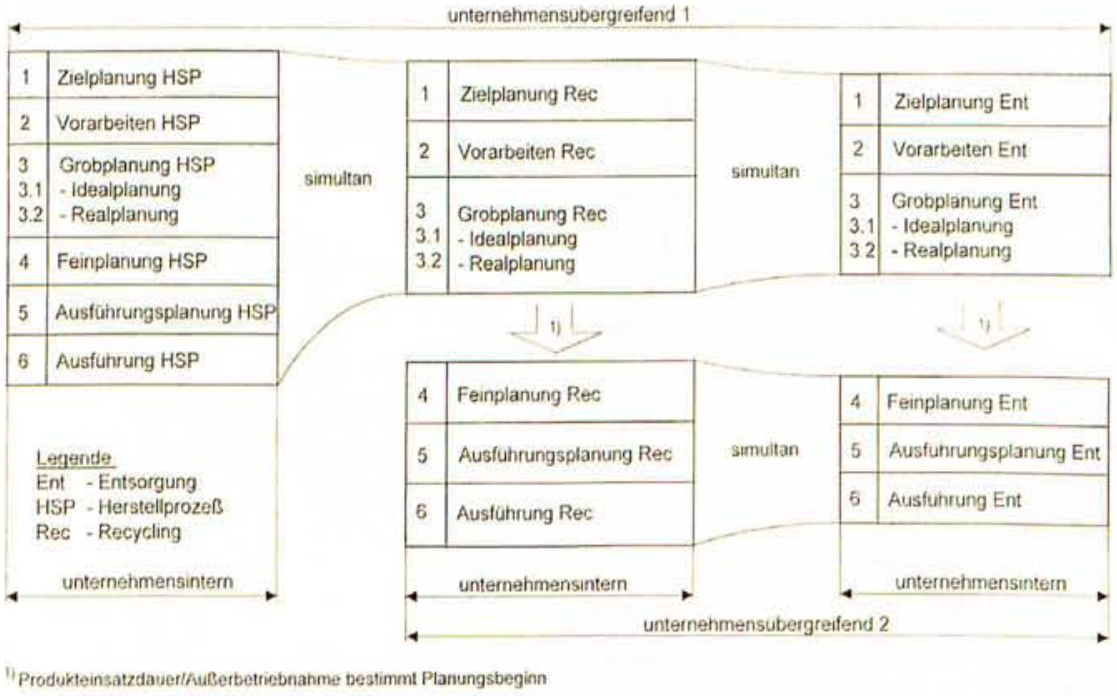

Bild 9: Unternehmensïbergreifende Verbundfabrikplanung - Herstellprozeß - Recycling - Entsorgeprozeß Beziehungen che interdisziplinäre Planungsteams sind willens und in der Lage, die umfangreichen und schwierigen Aufgabenkomplexe erfolgreich zu lösen. Während die Fabrikstrukturen fuir die Herstellungsprozesse bis hin zur Ausfuihrungsplanung zu entwerfen sind, bedarf es für die Recycling- und Entsorgungsprozesse einer zeitversetzten Bearbeitung entsprechend dem entscheidungsrelevanten Planungsfortschritt. Diese Neuorientierung der unternehmensübergreifenden Fabrikplanungsphilosophie zu einer entsprechenden durchgängigen Fabrikplanungsmethodik ist künftig immer umfassender durchzusetzen. 
Dem Anwender sind daher ablaufmethodisch strukturierte Inhalte dieser erweiterten Planungsmethodik zur Verfuigung zu stellen. Damit werden folgende Zielsetzungen verfolgt:

- Sicherung methodisch-inhaltlich rationeller Projektbearbeitungsprozesse im Rahmen von Fabrikplanungsvorhaben

- Schaffung von Grundlagen zum Entwurf komplexer Entwurfs- und Planungssoftware im stark erweiterten Methoden- und Objektbereich der Fabrikplanung.

Erste inhaltliche Ansätze einer solchen Planungsmethodik wurden vorgestellt.

\section{Literatur}

[1] Kettner, H.; Schmidt, J.; Greim, H.-R.:

Leitfaden der systematischen Fabrikplanung

Carl-Hanser-Verlag (1984)

[2] Grundig, C.-G.:

Fabrikplanung I - Grundlagen - (Lehrbrief)

Lehrbriefreihe Produktionsmanagement/Produktionslogistik Fernstudienagentur FHTW Berlin

1. Auflage (1996)

[3] Wirth, S.; Fischer, Th.:

Ein Weg zur Praktikablen ganzheitlichen Fabrikplanung

ZWF 89 (1994) $1 / 2$, S. 46-47

[4] Zülch, G.:

Integrierte Fabrikplanung

VDI-Zeitschrift 135 (1993) 3, S. 34-38

[5] Ahrend, H.-W.; Tabatzki, M.:

Teilefertigung und Montage durchgängig planen Werkstatttechnik (1993) 11/12, S. 86-89

[6] Baumgarten, H.:

Ganzheitliche Fabrikplanung

Wissenschaftliche Fachtagung, TU Berlin (25.01.1996)

[7] Wirth, S.; Baumann, A.:

Innovative Unternehmens- und Produktionsnetze

Wissenschaftliche Schriftenreihe TU Chemnitz Zwickau, Heft 8 (1996)

Institut für Betriebswirtschaften und Fabriksysteme (IBF)

[8] Demker, B.:

Durch kontinuierliche Verbesserung vom Teile- zum Modullieferanten

Zeitschrift für wirtschaftliche Fertigung 90 (1995) Nr. $1 / 2$, S. $18-20$

[9] Ahrend, H.-W.; Wagenhaus, G.:

Fertigungstiefe - Zulieferer - Umweltbelastung Aspekte zur unternehmensübergreifenden Fabrikplanung wt Produktion und Management 84 (1994) Heft 7/ 8 , S. 360-363

[10] Ahrend, H.-W.; Wagenhaus, G.: 